\title{
CLAREAMENTO PARA DENTES DESPOLPADOS: REVISÃO DE LITERATURA E CONSIDERAÇÕES
}

\section{BLEACHING OF PULPLESS TEETH: REVIEW OF LITERATURE AND CONSIDERATIONS}

\author{
Juliana Maria Capelozza Boaventura \\ Andiara Ribeiro Roberto* \\ João Paulo Martins de Lima* \\ Gislaine Cristina Padovani** \\ Luis César Brisighello**... \\ Marcelo Ferrarezi de Andrade ${ }^{* * n+\cdots}$
}

\begin{abstract}
RESUMO
A estética dos dentes não é privilégio dos dias atuais, pois preocupações nesse sentido têm sido relatadas desde 1860. Diante de um dente tratado endodonticamente e que apresenta qualquer tipo de escurecimento dental, o profissional pode lançar mão do clareamento intracoronário, que é a tentativa mais conservadora de restabelecer a cor normal dos dentes. O clareamento objetiva remover pigmentos por meio de agentes oxidantes e para isso foram desenvolvidas diversas técnicas. Entre os riscos do clareamento dental, o mais grave é a reabsorção cervical externa, que ocorre quando o produto clareador penetra através dos túbulos dentinários ao ligamento periodontal e inicia a reação inflamatória. Para evitar que ocorra tal reabsorção, é necessária a colocação de uma base protetora cervical. É importante conhecer o tempo que tem decorrido do tratamento clareador para o procedimento restaurador para se conseguir um ótimo selamento a fim de se evitar microinfiltração. Este estudo tem como objetivo revisar a literatura referente aos diversos tipos de agentes clareadores e os principais riscos adversos ao clareamento.

DESCRITORES: Clareamento dental • Dente não vital • Reabsorção da raiz • Peróxido de hidrogênio.
\end{abstract}

\section{ABS TRACT}

The esthetics of the teeth is not a privilege of our days, because concerns in that effect have been reported since 1860. Faced with an endodontically treated tooth that presents any type of color change, professionals can use intracoronary bleaching, which is more conservative attempt to restore the normal color of teeth. Bleaching aims to remove pigments by oxidizing agents and for that, various techniques were developed. Among the risks of tooth whitening the external resorption is the most severe, which occurs when the whitening penetrates through the dentin tubules to the periodontal ligament and initiates an inflammatory response. To prevent external resorption is required to place an intracoronal bleaching barrier. It is important to know the time between the bleaching treatment for restoring procedure for getting a good sealing to prevent microleakage. This study aims to review the literature about the different types of bleaching agents and their risks.

DESCRIPTORS: Tooth bleaching • Tooth, nonvital • Root resorption • Hydrogen peroxide.

* Mestre e Doutora em Ciências Odontológicas, área de concentração em Dentística Restauradora. Universidade Estadual Paulista Júlio de Mesquita Filho - UNESP, Araraquara-SP.

** Doutora em Ciências Odontológicas, área de concentração em Dentística Restauradora Universidade Estadual Paulista Júlio de Mesquita Filho- UNESP, Araraquara-SP

*** Mestre em Dentística Restauradora.Universidade Estadual Paulista Júlio de Mesquita Filho-UNESP, Araraquara-SP.

**** Mestre em Endodontia pela Universidade de São Paulo-USP, São Paulo-SP

***** Professor Doutor Livre-Docente do Departamento de Odontologia Restauradora. Universidade Estadual Paulista Júlio de Mesquita Filho- UNESP Araraquara-SP. 


\section{N T RODUÇÃO}

A estética dos dentes não é privilégio dos dias atuais, pois, desde 1860, preocupações nesse sentido têm sido relatadas na literatura odontológica, com a utilização das mais variadas substâncias (Ho e Goerig1, 1989, Haywood², 1992). A etiologia do escurecimento dos dentes despolpados é bem conhecida. Essa mudança de cor pode ser ocasionada por hemorragia advinda de trauma, técnica terapêutica inadequada, espaço de tempo entre o traumatismo e atendimento odontológico ou materiais obturadores e seladores dos canais radiculares contendo eugenol ou cones de prata (Lee et al. ${ }^{3}, 2004$ ).

Perante um dente tratado endodonticamente que apresenta qualquer tipo de escurecimento dental, o profissional pode lançar mão de alguns tipos de tratamento, como clarear o dente, recuperar a estética do dente através de manobras restauradoras ou protéticas, ou, ainda, associar as técnicas clareadoras com as restauradoras (Liebenberg 4 , 1997).

O clareamento é a tentativa mais conservadora de restabelecer a cor normal dos dentes e apresenta vantagens indiscutíveis tais como: evitar o desgaste da estrutura dentária em comparação com outros procedimentos, obter resultados estéticos satisfatórios comprovados em longo prazo, além de ser eficiente, relativamente simples e barato quando comparado ao tratamento protético (de Oliveira et al. ${ }^{5}, 2003$ ). É de suma importância que o profissional saiba diagnosticar adequadamente essas alterações de cor para indicar o melhor procedimento a ser utilizado ou até mesmo a associação de uma ou mais técnicas.

Sabe-se, portanto, que também há efeitos deletérios para os dentes e as estruturas de suporte, sendo a reabsorção cervical externa a mais grave descrita na literatura, e o primeiro relato foi descrito, por Harrington e Natkin ${ }^{6}$ (1979). Diferentes técnicas e materiais têm sido testados para evitar essa reabsorção. Autores (Friedman et al. ${ }^{7}, 1988$ ) recomendam o uso de material de clareamento em nível de epitélio juncional e a colocação de uma base protetora cervical.

Este estudo tem como objetivo revisar a literatura quanto aos diversos tipos de agentes clareadores e relatar os principais riscos adversos ao clareamento.

\section{REVISÃO DE LITERATURA}

Os primeiros relatos na literatura sobre o uso de agentes clareadores datavam desde 1860, e o uso das mais variadas substâncias foram propostas como: cloreto de cálcio, cloro, cloreto de alumínio, ácido oxálico, dióxido de enxofre, hipoclorito de sódio, entre outros. McQuillen ${ }^{8}$, em 1867, citava como agentes clareadores o dióxido de enxofre e o Licor de Labarraque (hipoclorito de sódio a 2,5\%), preconizado por Woodnutt ${ }^{9}$ (1860). Em 1877, Chapple $^{10}$ utilizava ácido oxálico para clarear dentes polpados e despolpados.

Já em 1895, Westlake ${ }^{11}$ descreveu a técnica de clareamento que utilizava pirozona (díóxido de hidrogênio), uma solução composta de cinco partes de peróxido de hidrogênio a $25 \%$ e uma parte de éter, mais corrente elétrica para acelerar a reação de liberação do oxigênio. O elétron positivo era colocado no dente e o negativo segurado pelo paciente.

$\mathrm{O}$ superoxol foi introduzido por $\mathrm{Ab}-$ bot $^{12}$ (1918). É uma solução de peróxido de hidrogênio a $30 \%$ por peso e $100 \%$ por volume, em água destilada. A solução embebida em algodão era aplicada na cavidade pulpar e o dente era aquecido através de uma lâmpada. Mas foi em 1924 que o uso de perborato de sódio começou a ser utilizado, e Prinz ${ }^{13}$ (1924) preconizou a técnica termocatalítica para dentes despolpados, onde utilizava uma solução saturada de perborato de sódio mais o peróxido de hidrogênio a $30 \%$ que eram preparados no momento do uso e, após serem aplicados no interior da câmara pulpar, eram ativados por uma fonte de calor.

Salvas ${ }^{14}$, em 1938, recomendava a utilização de uma pasta de perborato de sódio misturada com água destilada que era introduzida na câmara pulpar e mantida por vários dias. A grande vantagem dessa técnica é que o perborato de sódio fornece peróxido de hidrogênio (liberação de oxigênio) em meio alcalino, mas em contrapartida, apresenta menor velocidade de liberação de oxigênio.
BOAVENTURA JMC

ROBERTO AR

LIMA JPM

PADOVANI GC

BRISIGHELLO LC

ANDRADE MF

ClaREAMENTO

PARA DENTES

DESPOLPADOS:

REVISÃO DE

LITERATURA E

CONSIDERAÇÕES
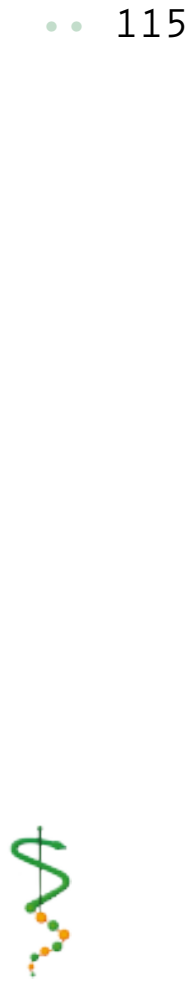

REV, ODONTOL,

UNIV, CID, SÃo

paulo

$2012 ; 24(2): 114-$

22, MAI $0-A G O$ 
BOAVENTURA JMC

ROBERTO AR

LIMA JPM

PADOVANI GC

BRISIGHELLO LC

ANDRADE MF

CLAREAMENTO

PARA DENTES

DESPOLPADOS:

REVISÃO DE

LITERATURA E

CONSIDERAÇÕES

\section{6}

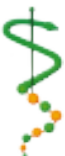

REV, ODONTOL.

UNIV, CID, SÃO

PAULO

2012; 24(2): 114 -

22, MAIO-AGO
Em 1946, Grossman ${ }^{15}$ propôs uma técnica de clareamento para dentes despolpados, utilizando peróxido de hidrogênio a 30\% mais o emprego de uma fonte de calor.

Spasser ${ }^{16}$ (1961) propôs uma técnica muito simplificada utilizando pasta de perborato de sódio e água destilada como veículo que depois de combinados para formar pasta, deveriam ser inseridos na cavidade pulpar. Essa técnica foi denominada Walking Bleach, já que o processo de clareamento ocorre entre as consultas, durante o período em que o agente clareador permanece selado na câmara pulpar.

Nutting e $\mathrm{Poe}^{17}$, em 1967, fizeram a modificação da técnica de Spasser utilizando o perborato de sódio mais o peróxido de hidrogênio, e indicavam o selamento do canal radicular com guta-percha antes de iniciar o tratamento. A pasta era aplicada dentro da câmara pulpar, permanecendo por 5 dias, quando então o paciente deveria voltar ao consultório. A troca de curativo deveria ser realizada quantas vezes fossem necessárias para a obtenção do clareamento. Essa técnica foi uma tentativa de potencializar o efeito do agente clareador, já que ambos apresentam capacidade de liberar oxigênio.

A variação dessa técnica usa gel de peróxido de hidrogênio a 35\% aplicado internamente à câmara pulpar e externamente à superfície vestibular do dente, com ativação da luz interna e externamente. A ativação da luz tanto interna quanto externamente pode ocorrer por fotopolimerização halógena convencional, lâmpada de arco de plasma ou o laser de diodo (Bartlett ${ }^{18}$, 2001).

Em 1997, a técnica de clareamento inside/outsite foi originalmente descrita na literatura americana por Settembrini et al. ${ }^{19}$ (1997) e a modificação dessa técnica foi descrita por Liebenberg 4 (1997). A técnica basicamente consiste na aplicação do peróxido de carbamida a $10 \%$ tanto na parte externa quanto na interna do dente de uma maneira simultânea, ou seja, a câmara pulpar não é vedada. Nessa técnica, utiliza-se uma moldeira que deve ser projetada de modo que haja um reservatório por palatino e vestibular para o dente em questão. Como nas outras técnicas, a câmara pulpar deve ser limpa, devendo haver a remoção da guta-percha de 2-3 mm abaixo da junção cemento-esmalte, para depois ser feito o tampão cervical. O agente clareador é aplicado no interior da cavidade e por vestibular, sendo mantido em posição com o auxílio da moldeira. A grande vantagem desses tratamentos é o curto período de tempo necessário para obtenção do efeito clareador e diminuição do risco de reabsorção cervical externa, devido à menor concentração do agente clareador e também porque não há necessidade da utilização de calor, o tempo de tratamento é menor e o oxigênio é completamente eliminado antes da confecção da restauração definitiva. Contudo, a manutenção da câmara pulpar aberta pode comprometer o selamento biológico promovido inicialmente pelo tratamento endodôntico e pelo tampão cervical, além de dificultar a higienização da cavidade pelo paciente (Liebenberg $\left.{ }^{4}, 1997\right)$.

A técnica imediata envolve a aplicação do condicionamento ácido tanto interna como externamente, com o objetivo de remover a smear layer e abrir os túbulos dentinários, aumentando, dessa forma, a permeabilidade do esmalte e dentina ao peróxido de hidrogênio. Esse procedimento tem sido considerado opcional devendo ser realizado apenas na primeira sessão do tratamento clareador. Alguns fabricantes de agentes clareadores não preconizam nem recomendam o condicionamento ácido dental (Andrade et al. ${ }^{20}$, 2005). O agente clareador recomendado para a técnica imediata é o peróxido de hidrogênio a 30-35\%, também utilizado para a técnica de clareamento em consultório para dentes com vitalidade pulpar. A manipulação do agente deve seguir as recomendações do fabricante e deve ser aplicado no interior da câmara pulpar e em toda a coroa dental externa, numa camada uniforme com espessura aproximada de $1 \mathrm{~mm}$. O gel deve descansar sobre o dente, por cerca de 2 minutos, para que haja penetração do peróxido de hidrogênio nos tecidos dentais. Depois de decorridos 2 minutos, o agente clareador deverá ser fotoativado com uma fonte de luz (Andrade et al. $\left.{ }^{20}, 2005\right)$. Quanto às fontes de luz empregadas, Carrasco et al. ${ }^{21}$ (2007) 
avaliaram a eficácia do peróxido de hidrogênio a 35\% para clareamento intracoronário com diferentes fontes de ativação de luz, como o LED e a lâmpada halógena, comparando com a técnica Walking Bleach e os resultados mostraram que foram similarmente eficazes.

\section{PRINCIPAIS RISCOS NO \\ CLAREAMENTO DE DENTES \\ DESPOLPADOS}

\section{Reabsorção cervical externa}

O mais importante e sério risco no clareamento de dente despolpado é a probabilidade de ocorrer reabsorção cervical externa (Harrington e Natkin ${ }^{6}, 1979$, Friedman et al. ${ }^{7}, 1988$, Ari e Ungor ${ }^{22}, 2002$, Heller et al. ${ }^{23}, 1992$, Trope $\left.{ }^{24}, 1997\right)$. Essa reabsorção de origem inflamatória é progressiva e ocorre abaixo da junção epitelial do dente. Dá-se através da perda de estrutura dentária radicular consequente da perda ou dano da camada de cementoblasto que ocorre devido à inflamação do tecido periodontal pelas substâncias cáusticas do clareamento que chegam à região periodontal pelos gaps na junção cemento-esmalte (Trope $\left.{ }^{24}, 1997\right)$.

Os primeiros relatos a sugerirem que os dentes clareados poderiam apresentar reabsorção externa após alguns anos de clareamento associada à utilização de fonte de calor ou a trauma dental prévio ao tratamento foram feitos por Harrington e $\mathrm{Na}$ tkin ${ }^{6},(1979)$. Os autores, através de sete casos clínicos, informaram que os pacientes apresentavam reabsorção no tempo de dois a sete anos após a realização de clareamento. Nesses casos, os pacientes eram jovens e todos apresentavam história de trauma anterior. Através desses acontecimentos postularam que o processo de reabsorção ocorreu pela difusão do agente clareador (Superoxol) através dos túbulos dentinários abertos para o ligamento periodontal cervical ou até mesmo pela injúria do periodonto pelo calor empregado para potencializar o agente clareador.

Após essa informação inicial de relatos de casos de reabsorção externa após clareamento dental, outros estudos surgiram. Em 1983, Lado et al. ${ }^{25}$, através de estudos com mulheres de 50 anos de idade, encontraram casos de reabsorção após clare- amento empregando a técnica termocatalítica e Walking Bleach. Mas seus estudos diferentemente de Harrington e Natkin ${ }^{6}$ (1979), não apresentavam história de trauma e os pacientes eram mais velhos. Para os autores, a técnica de clareamento desnatura a dentina exposta na linha cervical, onde existe um defeito entre o esmalte e o cemento, de tal forma que a dentina fica desprovida de cobertura cementária.

Então, de acordo com Harrignton e Natkin ${ }^{6}$, em 1979 a ocorrência de trauma previamente ao clareamento levava ao risco de reabsorção. No entanto, Lado et al..$^{25}$ (1983) descartaram essa possibilidade, uma vez que encontraram esse tipo de reabsorção em um dente sem história clínica de trauma.

Friedman et al.7 (1988), estudando 58 casos de dentes submetidos a tratamento clareador com peróxido de hidrogênio a $35 \%$ e calor, encontraram reabsorção em dois casos (3,5\%). Não houve história de trauma em qualquer um dos casos, e em um dos casos não houve emprego do calor. Os resultados desse trabalho reforçam as pesquisas de Lado et al..$^{25}$ (1983) e descartam a possibilidade de calor como fator etiológico da reabsorção relacionada ao clareamento.

Há especulação de que a difusão dos íons hidrogênio dos agentes para clareamento intracoronário possa produzir um ambiente ácido que é ótimo para atividade osteoclástica e reabsorção óssea, resultando em reabsorção cervical externa da raiz (Vaes ${ }^{26}$, 1968).

Dahlstrom et al. ${ }^{27}$ (1997) concluíram que os radicais hidroxilas são gerados durante o clareamento termocatalítico e essa geração de espécie química tóxica pode ser um mecanismo subjacente de destruição periodontal do tecido e reabsorção da raiz após clareamento intracoronário.

É importante que a cavidade coronária esteja limpa de resíduos e materiais endodonticos remanescentes, porque a presença de contaminantes na superfície pode influenciar negativamente a eficácia do agente clareador (Plotino et al. ${ }^{28}, 2008$ ).

\section{Diminuição da resistência à fratura}

Outro inconveniente é a queda da resistência que os dentes passariam a apre-
BOAVENTURA JMC

ROBERTO AR

LIMA JPM

PADOVANI GC

BRISIGHELLO LC

ANDRADE MF

CLAREAMENTO

PARA DENTES

DESPOLPADOS:

REVISÃO DE

LITERATURA E

CONSIDERAÇÕES

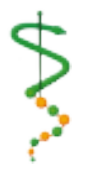

REV, ODONTOL.

UNIV. CID, São

PAULO

2012; $24(2)$ : $114-$

22, MAIO-AGO 
BOAVENTURA JMC

ROBERTO AR

LIMA JPM

PADOVANI GC

BRISIGHELLO LC

ANDRADE MF

CLAREAMENTO

PARA DENTES:

DESPOLPADOS:

REVISÃO DE

LITERATURA E

CONSIDERAÇÕES

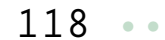

REV, ODONTOL.

UNIV, CID, SÃO

PAULO

2012; 24(2): $114-$

22, MAIO-AGO sentar, podendo levar à fratura. O tratamento clareador para estes dentes pode acrescentar uma somatória de efeitos comprometendo a sua resistência, principalmente quando há a perda das cristas marginais. Outro fator que deve ser levado em consideração é a falta de proteção em que o dente se encontra durante o período do tratamento clareador, pois o dente permanece oco, ou seja, sem material restaurador na câmara pulpar e, portanto, mais susceptível à fratura (Andrade et al. ${ }^{20}$, 2005).

Roberto $^{29}$ (2008) avaliou a influência de diferentes procedimentos restauradores na resistência à fratura de dentes tratados endodonticamente submetidos ao clareamento intracoronário e observou que os procedimentos restauradores com resina composta devolveram a resistência à fratura dos dentes tratados endodonticamente e submetidos ao clareamento dental interno, e que a associação da resina composta com pinos de fibra de vidro não aumentou a resistência à fratura em comparação ao uso isolado da resina composta.

\section{Alterações na adesividade}

Uma vez que o processo clareador é completado, restaurações estéticas podem necessitar de substituição a fim de se conseguir combinar ótima tonalidade. Alguns autores (Spalding et al. ${ }^{30}, 2003$, Marson et al. $\left.{ }^{31}, 2008\right)$ dizem que há efeitos prejudiciais na união dente-resina, e também na microinfiltração para os dentes restaurados após tratamento clareador. Esse efeito é atribuído à presença de peróxido residual ou oxigênio liberado dos agentes clareadores e mudanças estruturais na composição do esmalte e dentina que pode afetar o selamento da interface dente-resina (Barbosa et al. $.^{32}, 2008$ ).

Barbosa et al. ${ }^{32}$ (2008) avaliaram a influência do tempo de espera entre o término do clareamento dental e o processo restaurador, na força de adesão do esmalte e da dentina após o clareamento com peróxido de hidrogênio a 35\%. Os autores concluíram que os procedimentos restauradores adesivos no esmalte devem ter um intervalo de espera após o clareamento de sete dias, enquanto as restaurações em dentina devem ser feitas após 14 dias do clareamento dental.

Estudos mostraram que o clareamento com peróxido de carbamida a $10 \%$ e restaurado após 24 horas não originou diminuição da resistência adesiva após o clareamento, ao contrário do grupo clareado como peróxido de hidrogênio a 35\%, que provavelmente acorre por ser um gel de alta concentração e pela presença do oxigênio residual do material clareador que interfere na polimerização do sistema adesivo e da resina composta (Rotstein et al. $\left.{ }^{33}, 1992\right)$.

De acordo com Andrade et al. ${ }^{20}$ (2005), recomenda-se um tempo de espera de 7 a 10 dias para a realização da restauração, pois após esse período existe uma recuperação do potencial de adesão entre o substrato dental e substâncias adesivas. Existe outra consideração relacionada às propriedades ópticas do dente clareado, que se apresenta mais opacificado devido ao clareamento, podendo interferir na escolha da cor da restauração.

\section{INFLUENNCIA DOS TIPOS DE} AGENTES CLAREADORES

Uma das mais importantes propriedades de um material de clareamento é sua capacidade de permitir penetração do agente clareador através da permeabilidade dentinária. Carrasco et al. ${ }^{34},(2003)$, investigaram a eficácia de diferentes agentes clareadores internos na permeabilidade dentinária de dentes anteriores não vitais. Utilizaram o gel de peróxido de carbamida a 37\% (Whiteness Super-Endo), pasta de perborato de sódio preparada com 20\% de peróxido de hidrogênio (Merck Kgaa) e gel de peróxido de carbamida a $27 \%$ (preparado na escola farmacêutica da USP, Ribeirão Preto). A penetração dos íons cobre para os túbulos dentinários indicam que a permeabilidade dentinária foi alterada quando a estrutura dentária foi sujeita à ação dos agentes clareadores. A maior permeabilidade dentinária foi conseguida com o peróxido de carbamida a $37 \%$, seguida pela pasta de perborato de sódio/hidrogênio, sendo, portanto, o peróxido de carbamida a $27 \%$ o menos efetivo.

Lee et al. ${ }^{3}$ (2004) avaliaram as mudanças do $\mathrm{pH}$ e quantificaram a penetração do peróxido de hidrogênio usando três di- 
ferentes agentes clareadores intracoronários, o gel peróxido de carbamida a 35\%, o gel de peróxido de hidrogênio a $35 \%$ e perborato de sódio para clarear dentes humanos artificialmente escurecidos. Para o grupo-controle foi utilizada água. Durante a metodologia foram feitos quatro defeitos nos cementos preparados abaixo da junção cemento esmalte em cada superfície da raiz. Na presença de defeitos de cementos, concluíram que o peróxido de carbamida tem menores níveis de difusão extrarradicular do que o peróxido de hidrogênio, o que poderia ser uma boa alternativa para ser usado no clareamento intracoronário.

Em 2006, Oliveira et al. ${ }^{35}$ em seus estudos observaram que uma boa atividade antimicrobiana do agente clareador associado com um bom selamento coronário poderia prevenir uma microinfiltração de microrganismos dentro da obturação endodôntica, e por causa da sua atividade antibacteriana e substantividade, a clorexidina parece ter um bom potencial para aumentar as propriedades dos agentes clareadores. Eles associaram um gel base contendo clorexidina a $2 \%$ com um veículo de perborato de sódio e compararam a eficácia do perborato de sódio misturado com outros veículos, peróxido de carbamida a $37 \%$ e peróxido de hidrogênio a $30 \%$. Concluíram que o efeito de clareamento não diminuiu, mas a atividade antimicrobiana do gel de clorexidina deveria ser considerada boa alternativa para o veículo de perborato de sódio e como um suplemento para o gel de peróxido de carbamida, para prevenir uma possível microinfitração coronária.

Carrasco et al. ${ }^{21}$ (2007) avaliaram novamente a permeabilidade do agente clareador, o peróxido de hidrogênio a 35\%, só que ativado por fontes de luz como o LED e luz halógena e também pela técnica Walking Bleach e observaram que todas as três formas produziram aumentos similares na permeabilidade dentinária.

Valera et al. ${ }^{36}$ (2009), avaliaram a eficácia quanto ao poder de clareamento do gel de peróxido de carbamida a $16 \%$, perborato de sódio tetraidratado + água destilada e a mistura de peróxido de carbamida mais perborato de sódio. Como controle desse estudo foi utilizado um grupo sem produto clareador. Os autores observaram que os três agentes clareadores foram eficientes no clareamento intracoronario, especialmente após o período de 21 dias, mostrando que não houve diferença estatística, ao contrário dos autores Souza-Zaroni et al. ${ }^{37}$ (2009), que verificaram em seus estudos que a associação perborato de sódio/peróxido de carbamida a 37\% foi mais efetivo do que a associação perborato de sódio/água destilada.

\section{I SCUSSÃ 0}

O peróxido de hidrogênio penetra através dos túbulos dentinários para o ligamento periodontal e inicia uma reação inflamatória que resulta em reabsorção cervical externa. Rotstein ${ }^{38}$, (1991) relatou que a penetração do peróxido de hidrogênio foi significantemente mais alta em dentes onde havia defeitos no cemento e/ou no gap entre cemento e esmalte na junção cemento-esmalte. Isso, portanto, é agravado por falhas na junção amelocementária que propiciam uma margem de periodonto exposto. Além disso, a dissociação com calor leva a um aumento da reatividade do peróxido de hidrogênio, mas também um aumento da permeabilidade dentinária devido ao coeficiente de expansão térmica linear da dentina e um aumento das trincas que são notados nos casos de dentes traumatizados.

A pasta de hidróxido de cálcio usada como curativo após o uso dos agentes clareadores ajuda na prevenção da reabsorção cervical externa, pois tem uma capacidade tampão que evitaria a diminuição do Ph causado pelo agente clareador. O seu uso por 7 dias tem a finalidade de neutralizar o efeito dos agentes clareadores em toda a câmara pulpar e também ao nível cervical, pela capacidade da pasta em permear os tecidos dentais.

É também necessário conhecer o tempo decorrido do tratamento clareador ao procedimento restaurador para se conseguir um ótimo selamento, bem como reduzir o risco de microinfiltração nas restaurações adesivas (Shinohara et al. ${ }^{39}, 2001$ ).

Nos estudos de Shinohara et al. ${ }^{39}$ (2001), o aumento da microinfiltração após clareamento intracoronário de peró-
BOAVENTURA JMC

ROBERTO AR

LIMA JPM

PADOVANI GC

BRISIGHELLO LC

ANDRADE MF

CLAREAMENTO

PARA DENTES

DESPOLPADOS:

REVISÃO DE

LITERATURA E

CONSIDERAÇÕES

119
REV, ODONTOL.

UNIV. CID. SÃO PAULO

2012; $24(2)$ : $114-$ 22 , MAIO-AGO 
BOAVENTURA JMC :

ROBERTO AR

LIMA JPM

PADOVANI GC

BRISIGHELLO LC

ANDRADE MF

CLAREAMENTO

PARA DENTES:

DESPOLPADOS :

REVISÃO DE

LITERATURA E

CONSIDERAÇÕES

120

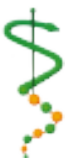

REV, ODONTOL,

UNIV. CID. SÃO

PAULO

$2012 ; 24(2): 114-$

22, MAIO-AGO xido de carbamida a $37 \%$ foi apenas detectado em margens dentinárias e não em margens em esmalte. Outros dois estudos $\left(\right.$ Crim $^{40} 1992$ e Ulukapi et al. ${ }^{41}$ 2003) informaram que as restaurações com resina composta após clareamento com peróxido de hidrogênio a $25 \%$ ou gel de peróxido de carbamida a $10-16 \%$ afetou adversamente o selamento marginal tanto das margens em dentina quanto às de esmalte.

Alguns autores (Titley et al.42, 1988, Torneck et al. ${ }^{43}$, 1991) têm sugerido que os efeitos deletérios do tratamento clareador poderiam ser causados pelo peróxido residual e oxigênio ativo, liberados pelos agentes clareadores, os quais inibem a polimerização de resina. Entretanto, tem-se mostrado que peróxido de hidrogênio a 30\% afetou os componentes orgânicos e inorgânicos da dentina, causando desnaturação de proteínas (Rotstein et al. ${ }^{33}$, 1992). Essas mudanças morfológicas poderiam reduzir o desempenho das restaurações de resina composta (Shinohara et al. $\left.{ }^{39}, 2001\right)$.

Mesmo com os possíveis riscos em se clarear dentes tratados endodonticamente, o clareamento pode ser executado com sucesso, desde que haja um diagnóstico correto, planejamento, técnica adequada empregada e controle do caso.

\section{CONSIDERAÇOES FINAIS}

Dentes escurecidos com tratamento de canal podem ser tratados com sucesso. Antes de realizar qualquer procedimento clareador em dentes é necessário verificar a qualidade do tratamento endondôntico. Várias técnicas foram propostas desde 1860 com diversos tipos de materiais para clareamento, sendo que a pasta de perborato de sódio mais o peróxido de hidrogênio se tornam mais efetivos do que quando o perborato é usado de forma isolada, porém apresenta maior risco com relação à reabsorção cervical externa. Esse risco aumenta quando há defeitos no cemento e/ou no gap entre a junção cemento/ esmalte. Então, para prevenir microcomunicação entre a câmara pulpar e a superfície externa da raiz, faz-se necessário utilizar uma base protetora de cimento de ionômero de vidro ou fosfato de zinco de $2 \mathrm{~mm}$ de espessura sob a junção cemento-esmalte.

É necessário conhecer o tempo decorrido do tratamento clareador ao procedimento restaurador para se conseguir um ótimo selamento, a fim de se evitar microinfiltração, pois estudos mostraram que efeitos deletérios do tratamento clareador poderiam ser causados pelo peróxido residual e oxigênio ativo liberados pelos agentes clareadores, inibindo a polimerização de resina. Portanto, é necessário esperar o prazo de 14 dias para se realizar o procedimento restaurador.

\section{REFERÊNCIAS}

1. Ho S, Goerig AC. An in vitro comparison of different bleaching agents in the discolored tooth. J Endod 1989 Mar;15(3):106-11.

2. Haywood VB. History, safety, and effectiveness of current bleaching techniques and applications of the nightguard vital bleaching technique. Quintessence Int 1992 Jul;23(7):471-88.

3. Lee GP, Lee MY, Lum SO, Poh RS, Lim KC. Extraradicular diffusion of hydrogen peroxide and $\mathrm{pH}$ changes associated with intracoronal bleaching of discoloured teeth using different bleaching agents. Int Endod J 2004 Jul;37(7):500-6.

4. Liebenberg WH. Intracoronal lightening of discolored pulpless teeth: a modified walking bleach technique. Quintessence Int 1997 Dec;28(12):771-7.

5. de Oliveira LD, Carvalho CA, Hilgert E, Bondioli IR, de Araujo MA, Valera MC. Sealing evaluation of the cervical base in intracoronal bleaching. Dent Traumato/ 2003 Dec;19(6):309-13. 
6. Harrington GW, Natkin E. External resorption associated with bleaching of pulpless teeth. J Endod 1979 Nov;5(11):344-8.

7. Friedman S, Rotstein I, Libfeld H, Stabholz A, Heling I. Incidence of external root resorption and esthetic results in 58 bleached pulpless teeth. Endod Dent Traumatol 1988 Feb;4(1):23-6.

8. McQuillen J. Bleaching discolored teeth. Dent Cosmos 1867 8(9):457.

9. Woodnutt C. Discoloration of dentine. Dent Cosmos 1860 2(12):662.

10. Chapple J. Restoring discolored teeth to normal. Dent Cosmos 1877 19(9):499.

11. Westlake A. Bleaching teeth by eletricy. Amer J Dental Science 1985 29(1):101.

12. Abbot C. Bleaching discolored teeth by means of the 30 per cent perhidrol and electric light rays. J Allied Dental Society 1918 13(3):259.

13. Prinz H. Recent improvements in tooth bleaching: a clinical syllabus. Dent Cosmos 1924 66(6):558-60.

14. Salvas J. Perborate as a bleaching agent. J Amer dent Assoc 1938 25(4):324.

15. Grossman L. Root canal therapy. Philadelphia: Lea \& Febiger; 1946.

16. Spasser H. A simple bleaching technique using sodium perborate. State Dent J 1961 27(9):332-4.

17. Nutting EB, Poe GS. Chemical bleaching of discolored endodontically treated teeth. Dent Clin North Am 1967 Nov;655-62.

18. Bartlett D. Bleaching discoloured teeth. Dent Update 2001 Jan-Feb;28(1):14-8.

19. Settembrini L, Gultz J, Kaim J, Scherer W. A technique for bleaching nonvital teeth: inside/outside bleaching. J Am Dent Assoc 1997 Sep;128(9):1283-4.

20. Andrade M, Huck C, Flores V. Clareamento dental: o clareamento à luz da ciência. In: Leonardo M. Endodntia: tratamento de canais radiculares, princípios técnicos e biológicos. São Paulo: Artes Médias; 2005. p. 1345-92.

21. Carrasco LD, Zanello Guerisoli DM, Pecora JD, Froner IC. Evaluation of dentin permeability after light activated internal dental bleaching. Dent Traumatol 2007 Feb;23(1):30-4.

22. Ari $\mathrm{H}$, Ungor M. In vitro comparison of different types of sodium perborate used for intracoronal bleaching of discoloured teeth. Int Endod J 2002 May;35(5):433-6.

23. Heller D, Skriber J, Lin LM. Effect of intracoronal bleaching on external cervical root resorption. J Endod 1992 Apr;18(4):145-8.

24. Trope M. Cervical root resorption. J Am Dent Assoc 1997 Apr;128 Suppl(56S-9S.

25. Lado EA, Stanley HR, Weisman MI. Cervical resorption in bleached teeth. Oral Surg Oral Med Oral Pathol 1983 Jan;55(1):78-80.

26. Vaes G. On the mechanisms of bone resorption. The action of parathyroid hormone on the excretion and synthesis of lysosomal enzymes and on the extracellular release of acid by bone cells. J Cell Biol 1968 Dec;39(3):676-97.

27. Dahlstrom SW, Heithersay GS, Bridges TE. Hydroxyl radical activity in thermo-catalytically bleached root-filled teeth. Endod Dent Traumato/ 1997 Jun;13(3):119-25.

28. Plotino G, Buono L, Grande NM, Pameijer CH, Somma F. Nonvital tooth bleaching: a review of the literature and clinical procedures. J Endod 2008 Apr;34(4):394-407.

BOAVENTURA JMC

ROBERTO AR

LIMA JPM

PADOVANI GC

BRISIGHELLO LC

ANDRADE MF

CLAREAMENTO

PARA DENTES

DESPOLPADOS:

REVISÃO DE

LITERATURA E

CONSIDERAÇÕES
REV, ODONTOL.

UNIV. CID, São

PAULO

2012; $24(2): 114-$

22, MAIO-AGO 
BOAVENTURA JMC

ROBERTO AR

LIMA JPM

PADOVANI GC

BRISIGHELLO LC

ANDRADE MF

CLAREAMENTO

PARA DENTES:

DESPOLPADOS :

REVISÃO DE

LITERATURA E

CONSIDERAÇÕES

29. Roberto A. Influência da aplicação de diferentes procedimentos restauradores na resistência à fratura de dentes submetidos ao clareamento dental interno com peróxido de hidrogênio a 38\% e fotoativação pelo sistema LED-laser [Dissertação]. Ribeirão Preto: Universidade de Ribeirão Preto; 2008.

30. Spalding M, Taveira LA, de Assis GF. Scanning electron microscopy study of dental enamel surface exposed to $35 \%$ hydrogen peroxide: alone, with saliva, and with 10\% carbamide peroxide. J Esthet Restor Dent 2003 15(3):154-64; discussion 65.

31. Marson FC, Sensi LG, Arruda T. Efeito do clareamento dental sobre a resistência adesiva do esmalte $R G O$ (Porto Alegre) 2008 jan.-mar. ;56(1):33-7.

32. Barbosa CM, Sasaki RT, Florio FM, Basting RT. Influence of time on bond strength after bleaching with 35\% hydrogen peroxide. J Contemp Dent Pract 2008 9(2):81-8.

33. Rotstein I, Lehr Z, Gedalia I. Effect of bleaching agents on inorganic components of human dentin and cementum. J Endod 1992 Jun;18(6):290-3.

34. Carrasco LD, Froner IC, Corona SA, Pecora JD. Effect of internal bleaching agents on dentinal permeability of non-vital teeth: quantitative assessment. Dent Traumatol 2003 Apr;19(2):85-9.

35. Oliveira DP, Gomes BP, Zaia AA, Souza-Filho FJ, Ferraz CC. In vitro assessment of a gel base containing $2 \%$ chlorhexidine as a sodium perborate's vehicle for intracoronal bleaching of discolored teeth. J Endod 2006 Jul;32(7):672-4.

36. Valera MC, Camargo CH, Carvalho CA, de Oliveira LD, Camargo SE, Rodrigues CM. Effectiveness of carbamide peroxide and sodium perborate in non-vital discolored teeth. J App/ Oral Sci 2009 May-Jun;1 7(3):254-61.

37. de Souza-Zaroni WC, Lopes EB, Ciccone-Nogueira JC, Silva RC. Clinical comparison between the bleaching efficacy of $37 \%$ peroxide carbamide gel mixed with sodium perborate with established intracoronal bleaching agent. Oral Surg Oral Med Oral Pathol Oral Radiol Endod 2009 Feb;107(2):e43-7.

38. Rotstein I. In vitro determination and quantification of $30 \%$ hydrogen peroxide penetration through dentin and cementum during bleaching. Oral Surg Oral Med Oral Pathol 1991 Nov;72(5):602-6.

39. Shinohara MS, Rodrigues JA, Pimenta LA. In vitro microleakage of composite restorations after nonvital bleaching. Quintessence Int 2001 May;32(5):413-7.

40. Crim GA. Prerestorative bleaching: effect on microleakage of Class V cavities. Quintessence Int 1992 Dec;23(12):823-5.

41. Ulukapi H, Benderli Y, Ulukapi I. Effect of pre- and postoperative bleaching on marginal leakage of amalgam and composite restorations. Quintessence Int 2003 Jul-Aug;34(7):505-8.

42. Titley KC, Torneck CD, Smith DC, Adibfar A. Adhesion of composite resin to bleached and unbleached bovine enamel. J Dent Res 1988 Dec;67(12):1523-8.

43. Torneck CD, Titley KC, Smith DO, Adibfar A. Effect of water leaching the adhesion of composite resin to bleached and unbleached bovine enamel. J Endod 1991 Apr;17(4):156-60.

Recebido em: 14/04/2011

Aceito em: 15/09/2011 